ANNALES

POLONICI MATHEMATICI

XXI (1969)

\title{
Analytic solutions of a linear functional equation
}

\author{
by M. KuczMa (Katowice)
}

In the present paper we are going to deal with the linear functional equation

$$
\varphi[f(z)]-g(z) \varphi(z)=h(z),
$$

where $\varphi(z)$ is the unknown function. We shall assume that the functions $f(z), g(z)$ and $h(z)$ are analytic in a neighbourhood of the origin, $f(0)=0$, $f^{\prime}(0)=0$ and $g(0)=0$. None of these functions is identically zero (the case of the homogeneous equation was already treated in [2]). Thus we may write

$$
\begin{array}{lll}
f(z)=z^{p} F(z), & F(0) \neq 0, & p \geqslant 2, \\
g(z)=z^{q} G(z), & G(0) \neq 0, & q \geqslant 1, \\
h(z)=z^{s} H(z), & H(0) \neq 0, & s \geqslant 0,
\end{array}
$$

where the functions $F(z), G(z)$ and $H(z)$ are analytic in a neighbourhood of the origin.

We shall be interested in local analytic solutions of equation (1) in a neighbourhood of the origin. A fundamental theorem concerning local analytic solutions of the functional equation

$$
\varphi(z)=h(z, \varphi[f(z)])
$$

has been given by W.. Smajdor [3] (cf. also [1]). Under the condition that $f(z)$ is analytic in a neighbourhood of a point $z=\zeta$ such that $f(\zeta)=\zeta$, and the function $h(z, w)$ is analytic in a neighbourhood of $(z, w)=(\zeta, \beta)$ such that $h(\zeta, \beta)=\beta$ and $0 \leqslant\left|f^{\prime}(\zeta)\right|<1$, every formal solution (1)

$$
\varphi(z)=\beta+\sum_{n=1}^{\infty} c_{n}(z-\zeta)^{n}
$$

of equation (5) is actual, i.e. has a positive radius of convergence.

(1) A formal solution of equation (5) is a formal power series that inserted into this equation satisfies it formally. 
In our case (cf. (2)) $\zeta=0$ and $f^{\prime}(\zeta)=f^{\prime}(0)=0$. If the function $h(z, w)$ in (5) is linear with respect to $w$ (with coefficients analytic in a neighbourhood of $z=0)$, then it is analytic in a neighbourhood of $(z, w)=(0, \beta)$ for every $\beta$. Moreover, for every $\beta$ fulfilling

$$
h(0, \beta)=\beta
$$

there exists exactly one formal solution

$$
\psi(z)=\beta+\sum_{n=1}^{\infty} c_{n} z^{n}
$$

of equation (5) (cf. [3]). Consequently, the result of W. Smajdor has the following consequence:

If $f(z)$ is analytic in a neighbourhood of the origin, $f(0)=f^{\prime}(0)=0$, and $h(z, w)$ is linear with respect to $w$ with coefficients analytic in a neighbourhood of the origin, then for every $\beta$ fulfilling (6) there exists exactly one solution $\varphi(z)$ of equation (5) that is analytic in a neighbourhood of the origin and fulfils the condition $\varphi(0)=\beta\left({ }^{2}\right)$.

However, we cannot apply this result directly to equation (1). Since $g(0)=0$, we cannot write equation (1) in form (5) with the right-hand side analytic in a neighbourhood of a point $(0, \beta)$. In the sequel we shall show how to overcome this difficulty and we shall reduce equation (1) to an equation of form (5) to which the result of W. Smajdor will be applicable.

Since $h(z) \not \equiv 0$, also $p(z) \neq 0$ for any solution of equation (1). Thus we may write

$$
\varphi(z)=z^{r} \Phi(z), \quad \Phi(0) \neq 0, \quad r \geqslant 0 .
$$

Inserting (2), (3), (4) and (7) into (1) we get

$$
z^{r p}\left[F^{\prime}(z)\right]^{\tau} \Phi[f(z)]-z^{q+r} G(z) \Phi(z)=z^{s} H(z) .
$$

The three terms in (8) have at the origin the zero of order $r p, q+r$ and $s$, respectively. Hence it follows that one of the following four cases must occur, provided that (1) has a solution (7):

$$
\begin{aligned}
& r p=q+r=s, \\
& r p=q+r<s, \\
& r p>q+r=s, \\
& r p=s<q+r .
\end{aligned}
$$

We shall consider the corresponding four cases separately.

(2) This solution can be obtained as the limit of successive approximations $\varphi_{n+2}(z)$ $=h\left(z, \varphi_{n}[f(z)]\right)$, where $\varphi_{0}(z)$ is an arbitrary function analytic in a neighbourhood of the origin and fulfilling the condition $\varphi_{0}(0)=\beta$. 
I. Calculating $r$ from (9) we get

$$
r=q /(p-1)
$$

and thus $q$ must be divisible by $p-1 ;(9)$ then becomes

$$
p q /(p-1)=s \text {. }
$$

We get from (8) and (9)

i.e.

$$
[F(z)]^{r} \Phi[f(z)]-G(z) \Phi(z)=H(z)
$$

$$
\Phi(z)=\frac{[F(z)]^{r}}{G(z)} \Phi[f(z)]-\frac{H(z)}{G(z)}
$$

This is an equation of form (5). Equation (6) takes the form

$$
G(0) \beta=[F(0)]^{r} \beta-H(0)
$$

and (since $H(0) \neq 0$ ) has a (unique) solution

$$
\beta=\frac{H(0)}{[F(0)]^{r}-G(0)}
$$

if and only if $G(0) \neq[F(0)]^{r}$. By W. Smajdor's theorem equation (15) has then a unique local analytic solution $\Phi(z)$ and consequently equation (1) has a local analytic solution

$$
\varphi(z)=z^{r} \Phi(z)=z^{q /(p-1)} \Phi(z) .
$$

Thus we arrive at the following result:

LEMMA 1. If $p-1$ divides $q$ and relation (14) holds and, moreover, $G(0) \neq[F(0)]^{q /(p-1)}$, then equation (1) has a local analytic solution in a neighbourhood of the origin. This solution is given by formula (16), where $\Phi(z)$ is the local analytic solution of equation (15).

II. Calculating $r$ from (10) we get (13) ( $q$ must again be divisible by $p-1$ ) and thus (10) becomes

$$
p q /(p-1)<s .
$$

Equation (8) now leads to

i.e.

$$
[F(z)]^{r} \Phi[f(z)]-G(z) \Phi(z)=z^{s-r p} H(z),
$$

$$
\Phi(z)=\frac{[F(z)]^{r}}{G(z)} \Phi[f(z)]-\frac{z^{s-r p} H(z)}{G(z)} .
$$

Equation (6) takes the form

$$
G(0) \beta=[F(0)]^{r} \beta .
$$


If $G(0)=[F(0)]^{r},(19)$ is fulfilled by every $\beta$. Applying W. Smajdor's theorem we obtain the following

LEMMA 2. If $p-1$ divides $q$ and relation (17) holds and, moreover, $G(0)=[F(0)]^{q /(p-1)}$, then equation (1) has a one-parameter family of local analytic solutions in a neighbourhood of the origin. These solutions are given by the formula

$$
\varphi(z)=z^{r} \Phi_{\beta}(z)=z^{q /(p-1)} \Phi_{\beta}(z),
$$

where, for every $\beta, \Phi_{\beta}(z)$ is the local analytic solution of equation (18) such that $\Phi_{\beta}(0)=\beta$.

If $G(0) \neq\left[F^{\prime}(0)\right]^{r}$, then $\beta=0$ is the only solution of (19). But, since $\beta=\Phi(0)$, we must have $\beta \neq 0$ according to (7). Of course, equation (18) has also a solution $\Phi_{0}(z)$ fulfilling $\Phi_{0}(0)=0$, but in the resulting formula for $\varphi(z)$ :

$$
\varphi_{0}(z)=z^{r} \Phi_{0}(z)
$$

$r$ is not the order of the zero of $\varphi$ at the origin. The actual order $r_{0}$ is greater than $r$ and thus it fulfils the inequality $r_{0} p>q+r_{0}$ (note that $p>1$ ). Thus we shall find the solution $\varphi_{0}(z)$ examining case (11) (cf. also the Remark after Lemma 3). Similarly, in Lemma 2 we should require $\beta \neq 0$.

III. Calculating $r$ from (11) we get

and (11) becomes

$$
r=s-q
$$

$$
p(s-q)>s
$$

Equation (8) leads to

i.e.

$$
z^{p r-s}[F(z)]^{r} \Phi[f(z)]-G(z) \Phi(z)=H(z),
$$

$$
\Phi(z)=\frac{z^{p r-s}[F(z)]^{r}}{G(z)} \Phi[f(z)]-\frac{H(z)}{G(z)}
$$

Equation (6) takes the form $\beta=-H(0) / G(0)$ and evidently has a unique solution. By W. Smajdor's theorem we obtain the following

LEMMA 3. If condition (21) is fulfilled, then equation (1) has a local analytic solution in a neighbourhood of the origin. This solution is given by

$$
\varphi(z)=z^{r} \Phi(z)=z^{\beta-q \Phi}(z),
$$

where $\Phi(z)$ is the local analytic solution of equation (22).

Remark. One should expect that several cases (9)-(12) can occur simultaneously, of course with different $r$. But it is readily seen that 
cases (9) and (10), (9) and (11), (9) and (12) exclude each other. If we had (10) and (12):

$$
r_{1} p=q+r_{1}<s \quad \text { and } \quad r_{2} p=s<q+r_{2},
$$

then $r_{2}<q /(p-1)=r_{1}<s / p=r_{2}$, a contradiction. Similarly, if we had (11) and (12):

$$
r_{1} p>q+r_{1}=s \quad \text { and } \quad r_{2} p=s<q+r_{2},
$$

then $r_{1}=s-q<r_{2}<q /(p-1)<r_{1}$, again a contradiction.

On the other hand, cases (10) and (11) may occur simultaneously. More than that, they must occur simultaneously whenever (10) occurs. For, if (10) occurs with an $r_{1}=q /(p-1)$, then (11) holds with $r_{2}=s-q$ $>r_{1}$ so that $p r_{2}>p r_{1}$. The solution obtained for $r_{2}$ is identical with the solution $\varphi_{0}(z)=z^{r_{1}} \Phi_{0}(z)$ excluded in the preceding case (because $G(0)$ $\neq[F(0)]^{r_{1}}$, or, in Lemma $\left.2, \beta=0\right)$.

1V. Calculating $r$ from (12) we get

$$
r=s / p
$$

and (12) becomes

$$
s(p-1)<p q
$$

Now we cannot write equation (8) in form (5) with the right-hand side analytic in a neighbourhood of a point $(0, \beta)$.

In view of (24) we have for $r$ given by (23) $r p<r+q$. But, since $p \geqslant 2$, there exists the smallest integer $R>r$ such that

Let us write

$$
R p \geqslant R+q \text {. }
$$

$$
\varphi(z)=P(z)+\varphi^{*}(z),
$$

where

$$
P(z)=\sum_{n=r}^{R-1} c_{n} z^{n}
$$

is a polynomial of a degree $<R$ and

$$
\varphi^{*}(z)=z^{\propto} \Phi^{*}(z), \quad \Phi^{*}(0) \neq 0, \quad \varrho \geqslant R .
$$

Inserting (26) into (1) we obtain

$$
\varphi^{*}[f(z)]-g(z) \varphi^{*}(z)=h^{*}(z),
$$

where

$$
h^{*}(z)=h(z)-P[f(z)]+g(z) P(z)
$$

Let us write

$$
h^{*}(z)=z^{\sigma} H^{*}(z), \quad H^{*}(0) \neq 0
$$


Equation (28) is quite analogous to (1). Since $\varrho \geqslant R$, it follows from (25) that $\varrho p \geqslant \varrho+q$. Consequently (9), (10) and (11) (with $r$ replaced by $\varrho$ and $s$ replaced by $\sigma$ ) are the only possible cases. At every case we must have

$$
\sigma \geqslant \varrho+q \geqslant R+q
$$

Relations (29), (30) and (31) are a key to determining polynomial (27). In fact, the condition that all the terms of degree $<R+q$ on the righthand side of (29) must vanish leads us to a system of $R+q-r$ equations with $R-r$ unknowns $c_{r}, \ldots, c_{R-1}$. In the expression $P[f(z)]$ the coefficient $c_{i}$ appears for the first time in the coefficient of $z^{i p}$, whereas in the expression $g(z) P(z)$ the $c_{i}$ appears for the first time in the coefficient of $z^{i+q}$. Since $R$ is the smallest integer fulfilling (25), we have $i p<i+q$ for $i=r, \ldots, R-1$. Consequently the condition that the coefficient of $z^{i p}$ must vanish has the form

$$
c_{i}[F(0)]^{i}+\left(\text { terms containing } c_{j} \text { with } j<i\right)=0 .
$$

In particular, for $i=r$ we obtain $c_{r}[F(0)]^{r}=H(0)$, whence $c_{r}=H(0)[F(0)]^{-r}$. After having determined $c_{j}$ for $j=r, \ldots, i-1$, we can determine $c_{i}$ uniquely from (32). However, since the number of equations is greater than the number of unknowns, it may happen that the $c_{i}$ found do not satisfy the remaining equations (those resulting by equating to zero the coefficients of $z^{n}$, where $n$ is not a multiple of $p$ ). Consequently we see that there may exist at most one polynomial (27) such that the function $h^{*}(z)$ given by (29) has at the origin a zero of order $\geqslant R+q$. If such a polynomial does not exist, equation (1) has no local analytic solution (26).

Now suppose that polynomial (27) exists. Then the problem of finding local analytic solutions of equation (1) reduces to that of finding local analytic solutions of equation (28), where the function $h^{*}(z)$ is well determined by (29). As have already remarked (cf. relation (31)), equation (28) may have local analytic solutions only in the three cases considered previously. Hence we obtain the following

LEMMA 4. Suppose that $p$ divides $s$ and relation (24) holds and that there exists a polynomial (27) (where $r$ and $R$ are determined by (23) and (25), respectively, $R$ being minimal) such that function (29) has at the origin a zero of order $\sigma \geqslant R+q$. If either

$$
\begin{aligned}
& \text { (i) } p-1 \text { divides } q, p q /(p-1)=\sigma \text { and } G(0) \neq[F(0)]^{q /(p-1)}, \\
& \text { or (ii) } p-1 \text { divides } q, p q /(p-1)<\sigma \text { and } G(0)=[F(0)]^{q /(p-1)}, \\
& \text { or } \\
& \text { (iii) } p(\sigma-q)>\sigma \text {, }
\end{aligned}
$$

then equation (1) has a local analytic solution

$$
\varphi(z)=P(z)+z^{e} \Phi^{*}(z)
$$


in a neighbourhood of the origin. Here $\varrho=q /(p-1)$ in cases (i) and (ii), $\varrho=\sigma-q$ in case (iii), and $\Phi^{*}(z)$ is the local analytic solution of the equation

$$
\Phi^{*}(z)=\frac{z^{\varrho p-q-\varrho}[F(z)]^{\varrho}}{G(z)} \Phi^{*}[f(z)]-\frac{z^{\alpha-q-\varrho} H^{*}(z)}{G(z)}
$$

in a neighbourhood of the origin. Solution (33) is unique in cases (i) and (iii) and depends on the parameter $\beta=\Phi^{*}(0)$ in case (ii).

It is clear from our considerations that the cases considered so far are the only ones where there may exist local analytic solutions of equation (1) in a neighbourhood of the origin. Thus we obtain the following

THEOREM 1. Suppose that the functions $f(z), g(z)$ and $h(z)$ are analytic in a neighbourhood of the origin, $f(0)=0, f^{\prime}(0)=0, g(0)=0$, none of the functions being identically zero. Then equation (1) has no local analytic solutions in a neighbourhood of the origin except for the cases covered by Lemmas 1 through 4.

The cases contained in Lemmas 1-4 are the only cases where equation (1) has formal solutions. In every such case we succeeded in reducing equation (1) to an equation of form (5), and then the theorem of W. Smajdor asserts that every formal solution is actual. Consequently we get the following

THEOREM 2. Under conditions of Theorem 1 every formal solution of equation (1) is actual, i.e. has a positive radius of convergence.

The assertion of Theorem 2 will no longer remain true if we drop the assumption $f^{\prime}(0)=0$. We shall return to this problem in a next paper.

\section{References}

[1] M. Kuczma, Une remarque sur les solutions analytiques d'une équation fonctionnelle, Coll. Math. 16 (1967), pp. 93-99.

[2] - Some remarks on a functional equation characterizing the root, Aequationes Math. (to appear).

[3] W. Smajdor, On the existence and uniqueness of analytic solutions of the functional equation $\varphi(z)=h(z, \varphi[f(z)])$, Ann. Polon. Math. 19 (1967), pp. 37-45. 